\title{
Mutations in the Liver Glycogen Synthase Gene in Children with Hypoglycemia due to Glycogen Storage Disease Type 0
}

\author{
Marju Orho, ${ }^{\star}$ Nils U. Bosshard, ${ }^{\circ}$ Neil R.M. Buist, ${ }^{\S}$ Richard Gitzelmann, ${ }^{\ddagger}$ Albert Aynsley-Green, Peter Blümel, \\ Mary C. Gannon, ${ }^{* *}$ Frank Q. Nuttall, ${ }^{* *}$ and Leif C. Groop* \\ *Department of Endocrinology, Wallenberg Laboratory, Malmö University Hospital, University of Lund, 20502 Malmö, Sweden; ${ }^{\ddagger}$ The \\ Division of Metabolic and Molecular Diseases, University Children's Hospital, University of Zürich, Zürich, Switzerland; ${ }^{\S}$ The \\ Department of Pediatrics and Medical Genetics, Oregon Health Sciences University, Portland, Oregon 97201; "Institute of Child Health, \\ Great Ormond Street Hospital for Children, London, United Kingdom; " Gottfried von Preyer'sches Kinderspital, Vienna, Austria; and \\ **Medicine and Food Science and Nutrition, University of Minnesota, Minneapolis, Minnesota 55417
}

\section{Abstract}

Glycogen storage disease type 0 (GSD-0) is a rare form of fasting hypoglycemia presenting in infancy or early childhood and accompanied by high blood ketones and low alanine and lactate concentrations. Although feeding relieves symptoms, it often results in postprandial hyperglycemia and hyperlactatemia. The glycogen synthase (GS) activity has been low or immeasurable in liver biopsies, whereas the liver glycogen content has been only moderately decreased. To investigate whether mutations in the liver GS gene (GYS2) on chromosome 12p12.2 were involved in GSD-0, we determined the exon-intron structure of the GYS2 gene and examined nine affected children from five families for linkage of GSD-0 to the GYS2 gene. Mutation screening of the 16 GYS2 exons was done by single-strand conformational polymorphism (SSCP) and direct sequencing. Liver GS deficiency was diagnosed from liver biopsies (GS activity and glycogen content). GS activity in the liver of the affected children was extremely low or nil, resulting in subnormal glycogen content. After suggestive linkage to the GYS2 gene had been established (LOD score $=2.9 ; P<$ 0.01), mutation screening revealed several different mutations in these families, including a premature stop codon in exon 5 (Arg246X), a 5'-donor splice site mutation in intron 6 $\left(\mathrm{G}^{+1} \mathrm{~T} \rightarrow \mathrm{CT}\right)$, and missense mutations Asn39Ser, Ala339Pro, His446Asp, Pro479Gln, Ser483Pro, and Met491Arg. Seven of the affected children carried mutations on both alleles. The mutations could not be found in $\mathbf{2 0 0}$ healthy persons. Expression of the mutated enzymes in COS7 cells indicated severely impaired GS activity. In conclusion, the results demonstrate that GSD-0 is caused by different mutations in the GYS2 gene. (J. Clin. Invest. 1998. 102:507-515.) Key words: glycogen synthase deficiency $\bullet$ childhood hypoglycemia • ketotic hypoglycemia • postprandial hyperglycemia • glycogen storage

Address correspondence to Leif Groop, M.D., Professor, Department of Endocrinology, Malmö University Hospital, Wallenberg Laboratory, plan 3, 20502 Malmö, Sweden. Phone: 46-40-337-217; FAX: 46-40-337-042; E-mail: Leif.Groop@endo.mas.lu.se

Received for publication 22 January 1998 and accepted in revised form 15 May 1998.

J. Clin. Invest.

(C) The American Society for Clinical Investigation, Inc. 0021-9738/98/08/0507/09 \$2.00

Volume 102, Number 3, August 1998, 507-515

http://www.jci.org

\section{Introduction}

Hypoglycemia in children associated with hepatic glycogen synthase $(\mathrm{GS})^{1}$ deficiency is a seemingly rare disorder also referred to as glycogen storage disease type 0 (GSD-0) (1-4). Patients present in infancy or early childhood with fasting hypoglycemia accompanied by high levels of blood ketones and low levels of alanine and lactate (1-3). Feeding relieves symptoms and reverses the abnormal biochemical profile often resulting in postprandial hyperglycemia and hyperlactatemia. The GS activity has been low or immeasurable in liver biopsies, whereas the liver glycogen content has been decreased only moderately, suggesting some residual glycogen synthesis (1-3).

Although the disease seems to be inherited in an autosomal recessive fashion (1-3), the underlying molecular defects have not been known. GS deficiency could be the consequence of defective expression or activation of the GS or synthesis of a defective protein. Mice lacking the transcription factor CCAAT/enhancer-binding protein $\alpha(C / E B P \alpha)$ gene (5-7) do not store liver glycogen normally and they die from hypoglycemia (8). Using polymorphic microsatellite markers flanking the $C / E B P \alpha$ gene on human chromosome 19, we could exclude linkage between this chromosomal region and GSD-0. Furthermore, mixing the liver homogenates from affected and unaffected individuals did not activate the GS (2). Therefore, available data point at an inherited defect in the GS enzyme.

To examine whether mutations in the liver GS gene (GYS2) were involved in GSD-0, we determined the exon-intron structure of the GYS2 gene and studied nine affected children from five families for linkage using intragenic and flanking polymorphic markers. After linkage to the chromosomal region 12p12.2 (9) had been established, we screened the coding regions, the exon-intron boundary regions, and part of the putative promoter of the GYS2 gene for mutations. Mutations were found in all affected children, indicating that GSD-0 is caused by molecular defects in the GYS2 gene.

\section{Methods}

\section{Patients}

Patient 1 (family Y). Male child D.Y. was born in 1988 to unrelated Turkish parents living in Austria. He had three seemingly healthy brothers. He was referred at age 4 because of short stature. Height

1. Abbreviations used in this paper: G-6-P, glucose-6-phosphate; GS, glycogen synthase; GSD-0, glycogen storage disease type 0; GYS2, liver GS gene; SSCP, single-strand conformational polymorphism; UDPG, UDP glucose. 


\begin{tabular}{|c|c|c|c|c|c|c|}
\hline & \multicolumn{5}{|c|}{ Patient number } & \multirow[b]{2}{*}{ Controls } \\
\hline & 1 & 2 & 5 & 6 & 8 & \\
\hline & & & & & & $n=13$ \\
\hline Glycogen ( $\mathrm{g} / 100 \mathrm{~g}$ of liver $)$ & 2.5 & 0.8 & $0.7 ; 1.7$ & 1.2 & 0.65 & $2.4-6.4$ \\
\hline Absorption maximum of the glycogen-iodine complex $(\mathrm{nm})$ & 471 & ND & ND & 471 & 468 & $454-478$ \\
\hline \multicolumn{7}{|l|}{ GS activity $(\mu \mathrm{mol} / \mathrm{g} \times \min )$} \\
\hline with G-6-P & 0.12 & ND & $0.0 ; 0.04$ & 0.0 & 0.04 & $1.1-5.5$ \\
\hline without G-6-P & 0.02 & ND & $0.0 ; 0.02$ & 0.0 & $<0.01$ & $0.03-0.6$ \\
\hline
\end{tabular}

$N D$, Not done.

was $86.5 \mathrm{~cm}(-3 \mathrm{SD})$ and weight was $11.4 \mathrm{~kg}(-3 \mathrm{SD})$. Serum growth hormone concentration was subnormal $(5.1 \mathrm{ng} / \mathrm{ml})$. After an overnight fast, the blood glucose was $1.7 \mathrm{mmol} /$ liter, whereas free fatty acids and $\beta$-hydroxybutyrate concentrations were high. In relation to hypoglycemia, the plasma insulin levels were appropriately reduced and cortisol, lactate, pyruvate, and alanine concentrations were normal. An oral glucose tolerance test $(1.75 \mathrm{~g} / \mathrm{kg}$ body wt) provoked an excessive rise of blood glucose $(10.1 \mathrm{mmol} / \mathrm{liter})$ and lactate $(7.0$ $\mathrm{mmol} /$ liter), whereas free fatty acids and $\beta$-hydroxybutyrate were normal. A similar metabolic profile was seen $1 \mathrm{~h}$ after a meal. At age 7, a liver biopsy showed low GS activity with glycogen content in the low normal range (Table I). He tolerated fasting periods of $5 \mathrm{~h}$, refused uncooked cornstarch (3), but was given a midnight meal instead. His mental development was normal.

Patient 2 (family M). Female child L.M. was born in 1987 in Oregon. At age 5 she presented with fasting hypoglycemia and postprandial hyperglycemia. Her blood glucose levels rose from 2.7 to 22 $\mathrm{mmol} /$ liter after feeding. The glycogen content in a liver biopsy was low (Table I), whereas the activities of glucose-6-phosphatase, debranching enzyme, total and active phosphorylase, fructose-1,6-bisphosphatase, and fructose aldolase as well as the glucose-1-phosphate/ glucose ratio were all normal. GS activity was not measured, but the clinical picture together with the biochemical findings suggested GSD-0. Symptoms of hypoglycemia were prevented by ingestion of cornstarch. Her mental development has been slow. Her mother also develops hypoglycemia during prolonged fasting.

Patient 3 (family $M$ ). Male child S.M., born in 1989, was the younger brother of patient 2 . At age 3 , he developed symptoms of fasting hypoglycemia and postprandial hyperglycemia. No liver biopsy was performed but he was suspected to have the same disorder as his sister. Cornstarch feeding prevented hypoglycemia. His mental development was normal.

Patient 4 (family M). Female child M.M., born in 1989, was the younger sister of patient 2 . Her clinical picture was similar to her sister and she was suspected of having GSD-0 at the age of $1 \mathrm{yr}$. No liver biopsy was performed. Cornstarch feeding prevented the worst episodes of hypoglycemia. Her mental development was normal.

Patient 5 (family F). Female child I.F. was born in 1987 to unrelated German parents. At age 3.5 she was drowsy in the mornings, occasionally vomited, and her pediatrician discovered hypoglycemia and marked ketonuria. At age 4 she was suspected of having liver disease. Metabolic profiles were indicative of GSD-0. Glycogen content in the liver was low and GS activity was virtually absent (Table I) (3).

Patient 6 (family J). Male child D.J. was born in 1991 to unrelated German parents. At age 2 he appeared tired in the mornings and uninterested in his breakfast. He recovered after eating. At age 3.5 his pediatrician diagnosed hypoglycemia and ketonuria. Metabolic profiles were indicative of GSD-0. The diagnosis of hepatic GS deficiency was confirmed by a liver biopsy, which demonstrated low glycogen content and GS deficiency (Table I) (3).

Patient 7 (family J). M.J., born in 1989, was the older brother of patient 6 . Metabolic profiles indicative of GSD-0 were observed but no liver biopsy was performed (3).

Patient 8 (family S). Female child K.S. was born in 1966 to unrelated British parents. With the establishment of daytime feeding, early morning behavioral changes, drowsiness, and lack of attention was noted. Symptoms were reversed by food intake. At $7 \mathrm{yr}$ of age she had occasional morning convulsions. Fasting hypoglycemia and ketonuria were discovered and GS deficiency was diagnosed by a liver biopsy (Table I) $(2,10)$. At age 29 she gave birth to a healthy child (11).

Patient 9 (family S). D.S., born in 1964, was the older brother of patient 8 . When he was old enough to go through the night without feeding, he became drowsy and unresponsive in the morning until the first meal. Symptoms ceased after 3 yr of age. At age 13 he was examined together with his sibling and the metabolic profiles resembled those of his sister with diagnosed GSD-0 (12). A liver biopsy was not performed.

\section{Liver biopsies and biochemical analyses}

In five children, open or percutaneous liver biopsies were performed during infusion of $10 \%$ glucose after the children had fasted for at least $4 \mathrm{~h}$. Liver biopsies taken from cadaveric kidney donors with parenteral nutrition served as controls (3). The specimens were frozen immediately and kept at $-20^{\circ} \mathrm{C}$ until analyzed for glycogen (13), glycogen-iodine complex (14), and GS (EC 2.4.1.11) activity (3).

\section{Isolation and partial sequence of the human GYS2 gene}

A human placental genomic library in phage (catalog No. 946205; Stratagene, La Jolla, CA) was screened with $\left[\alpha-{ }^{32} \mathrm{P}\right] \mathrm{dCTP}-$ labeled (Pharmacia Biotech, Uppsala, Sweden) 2.1-kb long GYS2 cDNA probe (15) containing the whole coding sequence and with a PCR probe containing only the first $441 \mathrm{bp}$ of the cDNA. The probes were labeled to a specific activity of $>10^{8} \mathrm{cpm} / \mu \mathrm{g}$ DNA using the Readyto-go DNA labeling kit (Pharmacia Biotech). Labeled probes were purified in Sephadex-G50 nick-columns (Pharmacia Biotech). Several genomic clones containing parts of the GYS2 gene were isolated and five of them (GYS2- $\lambda$ E1-3, GYS2- $\lambda 6$, GYS2- $\lambda 14$, GYS2- $\lambda 16$, and GYS2- $\lambda 21$ ) were chosen for further analysis. The genomic clone GYS2- $\lambda E 1-3$ was extracted when the genomic library was screened with the 441-bp PCR probe, whereas the other clones were extracted with the $2.1-\mathrm{kb}$ cDNA probe. These clones were digested with several restriction enzymes, run on an agarose gel, and blotted on a nylon filter. The blots were then probed with a 2.1-kb GYS2 cDNA probe to identify the fragments containing exons. DNA fragments carrying exons and the 5'-flanking region were subcloned into a pGEM4Z vector (Promega, Madison, WI) or amplified by PCR either from genomic or genomic clone DNA. All the exons, exon-intron boundaries, and the $5^{\prime}$-flanking region were sequenced bidirectionally by the dideoxy chain termination method using either a Sequenase 2.0 sequencing kit (U.S. Biochemicals, Cleveland, OH) or an ABI PRISM dye terminator cycle sequencing ready reaction kit (Perkin Elmer, Foster City, 
CA) and automated sequencer analysis (ABI, model 373; Perkin Elmer). The intron sizes were determined by Southern blotting, restriction mapping, and/or sequencing of the PCR products. Each clone containing part of the GYS2 gene was also screened for microsatellite markers by hybridization of the Southern blots containing restriction enzyme-digested GYS2 genomic clone DNA with a degenerated $\left[\alpha{ }^{32} \mathrm{P}\right] \mathrm{dCTP}-$ labeled CA/GT - probe. Positive fragments were subcloned into a pGEM $4 \mathrm{Z}$ vector and sequenced.

\section{Linkage analysis}

Linkage to the GYS2 gene was tested using an intragenic microsatellite marker GYS2-CA, which we identified in the gene and flanking markers GATAglH01 (16), D12S1606 (17), and D12S1688 (17). The affected individuals and their family members were haplotyped using radioactive PCR followed by fragment separation on a 5\% denaturing polyacrylamide gel. For GYS2-CA, $30 \mathrm{ng}$ of genomic DNA was amplified using 3 pmol of $\left[\gamma^{-32} \mathrm{P}\right] \mathrm{dATP}$ end-labeled oligonucleotide primer GYS2-CAF (5'-TTCGATAGTGTAATCTACAGC) and 3 pmol of unlabeled primer GYS2-CAR (5'-TGGTATGTAGTAACATTAACAC) with $0.5 \mathrm{U}$ Taq polymerase (Perkin Elmer) in $1 \times$ ammonium sulfate buffer ( $16 \mathrm{mM}$ ammonium sulfate; $67 \mathrm{mM}$ Tris, $\mathrm{pH} 8.8 ; 0.01 \%$ Tween), $1.5 \mathrm{mM}$ magnesium chloride, $200 \mu \mathrm{M}$ of the deoxynucleotide triphosphatases, and $2 \%$ formamide. PCR reactions were performed in Gene Amp PCR System 9600 (Perkin Elmer) with initial denaturation $\left(5 \mathrm{~min}\right.$ at $\left.94^{\circ} \mathrm{C}\right)$, followed by 30 cycles of denaturation $\left(30 \mathrm{~s}\right.$ at $\left.94^{\circ} \mathrm{C}\right)$, annealing $\left(30 \mathrm{~s}\right.$ at $\left.56^{\circ} \mathrm{C}\right)$, and extension $(30 \mathrm{~s}$ at $\left.72^{\circ} \mathrm{C}\right)$ and by final extension $\left(10 \mathrm{~min}\right.$ at $\left.72^{\circ} \mathrm{C}\right)$. The earlier described PCR conditions were used for markers GATAglH01, D12S1606, and D12S1688 $(16,17)$. An autosomal recessive inheritance with $100 \%$ penetrance was assumed and the LOD score calculated (all five families pooled for the analysis) using the GENEHUNTER 1.0 program (18).
Single-strand conformational polymorphism (SSCP) analysis

For the SSCP analysis (19), $50 \mathrm{ng}$ of genomic DNA extracted from each affected individual, their family members, and at least two healthy control subjects was amplified with 10 pmol each of the GYS2 gene-specific intronic primer pairs (Table II). The PCR was carried out in $20-\mu \mathrm{l}$ reactions using $0.5 \mathrm{U}$ of Taq polymerase and including $0.05 \mu \mathrm{l}$ of $\left[\alpha_{-}{ }^{32} \mathrm{P}\right] \mathrm{dCTP}(3,000 \mathrm{Ci} / \mathrm{mmol})$ (Pharmacia Biotech). Reactions were diluted $1: 1$ with $95 \%$ formamide buffer, denatured 5 min at $94^{\circ} \mathrm{C}$, cooled, and electrophoresed on a gel with $5 \%$ glycerol $(8 \mathrm{~W}$ for $13 \mathrm{~h}$ at room temperature) and on a glycerol-free ( $35 \mathrm{~W}$ for $4 \mathrm{~h}$ at $\left.4^{\circ} \mathrm{C}\right)$ nondenaturing $5 \%$ polyacrylamide gel (49:1 acrylamide/bisacrylamide). When shifts in the band pattern were observed, the corresponding exon was PCR amplified from genomic DNA, isolated from an agarose gel, and sequenced bidirectionally using the same primers as for SSCP.

\section{Confirmation of mutations by restriction enzyme digest}

All the identified missense mutations and the intron 6 splicing site mutation were confirmed by PCR of the genomic DNA followed by restriction enzyme digestion and agarose gel electrophoresis in all affected subjects, the studied family members, and in 200 healthy control subjects. The missense mutations Ala339Pro, His446Asp, and Met491Arg were confirmed by PCR with the primer pairs used in the SSCP followed by digestion with restriction enzymes AluI, BsEDI, and BstNI, respectively (New England Biolabs, Beverly, MA). For detection of intron $6\left(\mathrm{G}^{+1} \mathrm{~T} \rightarrow \mathrm{CT}\right)$, Asn39Ser, Pro479Gln, and Ser483Pro mutations, which did not create or destroy a known restriction enzyme recognition site, the PCR from genomic DNA was performed using one of the SSCP primers (Table II) and a new primer where a single nucleotide mismatch was introduced (the mismatch nucleotide is underlined) to create an AluI (E6-GT/CT-F, 5'-GATTTTGTTCGAGGTCATTTCTATAG with primer E6R; for

Table II. Oligonucleotide Primer Pairs Used to Amplify the GYS2 Gene

\begin{tabular}{|c|c|c|c|c|}
\hline Exon & Upstream primer sequence & Downstream primer sequence & Size of PCR product & Annealing temperature \\
\hline & & & $b p$ & ${ }^{\circ} \mathrm{C}$ \\
\hline 1 & GGAGGACTGTAAGAAGAATG & CTACATTCCTCAGTCACCTAG & 194 & 56 \\
\hline 2 & ATTGCTAACCTGAATGATAATAG & AAGTTTACTGAGAATTGCCAAG & 268 & 58 \\
\hline 3 & TCTTGCATGACTTAATATCACTAC & GTTGTGCTGCTCCTCCGTTG & 283 & 62 \\
\hline 4 & ATCGACAACTTTTCTGAATG & GCTTCAGCAATCTGAAAGAAGG & 278 & 52 \\
\hline 5 & AGGGCCATTGTTAGCAAATGTG & GTAACATCATTCGGAACTGAAAG & 239 & 62 \\
\hline 6 & TTCTACAATGGTACCCTCTTTG & CAAAGATCACTCATATCTGATAC & 234 & 58 \\
\hline 7 & TTGTTACTGTTGCTGTATTTCAT & GAAGAAAGTTCTCCCTACAAG & 226 & 56 \\
\hline 8 & AATGTTTAAGTTCCATCTATCATAC & GCGATACATGAGATGTCATTCAC & 262 & 62 \\
\hline 9 & AAGATACCTGTAATTTCAAAG & TTAATAAGTTATGATGTCTAAATTG & 253 & 54 \\
\hline 10 & ATCTGATGCTTAGGAATCACTG & AATGTTCCAAATTAGCACATTCC & 245 & 60 \\
\hline 11 & GAAATGTCAATGACCTATTGTTG & CTAAGGAAAGCTAATAAATTCTCAG & 275 & 56 \\
\hline 12 & TACTGAAATAACCAAATGTAGGAG & CAAAATTCAAAACATTCCACACTC & 250 & 62 \\
\hline 13 & TATAGGCGCTGGTCTGAAGCC & TGTTTGGTTAGAGGTCATGTCTC & 197 & 60 \\
\hline 14 & GAACAAATGTTTAAGTTAGTCCTAC & GAATCAATATGTTATAGTCCAGTGG & 289 & 62 \\
\hline 15 & TAACTTGATTATTGAGATATCTAAGG & GCTTGTGTGCATAGCTACTTCC & 236 & 62 \\
\hline $16 \mathrm{~A}$ & GAAAGCCAAGAAACTGTTGTGATG & GAGGACTGGAGGCCTGAGAC & 123 & 62 \\
\hline $16 \mathrm{~B}$ & AGTACCACCTTCTCCTTCAG & AAATGAAATTTGTGGCATT & 251 & 56 \\
\hline $5^{\prime}-\mathrm{A}$ & ATTTCATGCTACTAGTTTATTCC & GAAGCCCACCCAGGGATGTTACAG & 195 & 56 \\
\hline $5^{\prime}-\mathrm{B}$ & CCTATCCCAGGAAGTGCTTACC & ACCAGGCTTTGGTAGCTTCTC & 226 & 62 \\
\hline $5^{\prime}-\mathrm{C}$ & TGTAATCTAAGGAGGCCTGC & AGAAAAATCCTCTGATTCAGGAC & 198 & 58 \\
\hline
\end{tabular}

Primers for amplifying exons 2-15, the downstream primer for exon 1, and the upstream primer for exon 16 (16A) are designed from intronic regions. The upstream primer for exon 1, the downstream primer for exon 16, (16A) and the primers for amplifying exon 16B are designed from the GYS2 cDNA (15). Exons 16A and 16B amplify the coding regions of exon 16 (nucleotides 1919-1991 and 1971-2222, respectively). 5'-A amplifies the first 74 nucleotides of exon 1 and the first 121 nucleotides upstream of the $5^{\prime}$-nontranscribed region. 5'-B and 5'-C amplify fragments from the $5^{\prime}$-region (nucleotides -74 to -300 and -252 to -450 , respectively). 
detection of intron 6 mutation), a SpeI (E1-39Ser-F, 5' -TTGAAGTTGCTTGGGAAGTGACTA with primer E1R; for detection of the Asn39Ser mutation), a BstNI (E12-479Gln-R, 5'-GGACTGGTGGAGGATAGAAACCCT with primer E12F; for detection of the Pro479Gln mutation), or an RsaI (E12-483-F, 5'-GTGATTTTGCACCCAGAGTTTGTA with primer E12R; for detection of the Ser483Pro mutation) recognition site in case of a mutated sequence. The digestions were performed according to manufacturer's instructions (New England Biolabs) and the digested PCR products were resolved on $4.5 \%$ agarose gel with ethidium bromide $(0.3 \mu \mathrm{g} / \mathrm{ml})$ and visualized under ultraviolet light.

\section{Site-directed mutagenesis}

Site-directed mutagenesis of the GYS2 cDNA was performed using QuickChange mutagenesis kit with Pfu-polymerase (Stratagene). The GYS2 cDNA (nucleotides 1-2140) was cloned to the pcDNA3.1- expression vector (In Vitrogen, NV, Leek, The Netherlands) and named GYS2WT-pcDNA3.1-. The following complementary mutagenic primers were used to mutagenize the GYS2WT-pcDNA3.1- (with mutagenized base underlined): $\left(5^{\prime} \rightarrow 3^{\prime}\right)$ 39SerF, GCTTGGGAAGTGACCAGTAAAGTTGGAGGCATC, 39SerR, GATGCCTCCAACTTTACTGGTCACTTCCCAAGC, 246XF, CGGTACTGCATGGAGTGAGCTTCCGTTCATTGC, 246XR, GCAATGAACGGAAGCTCACTCCATGCAGTACCG, 339ProF, GAGTTTTCAAACAAAGGACCTGACATCTTCCTAG, 339ProR, CTAGGAAGATGTCAGGTCCTTTGTTTGAAAACTC, 446AspF, CCCCAGTGACCACGGACAACATGATTGATGAC, 446AspR, GTCATCAATCATGTTGTCCGTGGTCACTGGGG, 479GlnF, CAAGGTGATTTTGCACCÄAGAGTTTCTATCCTCC, 479GlnR, GGAGGATAGAAACTCTTGGTGCAAAATCACCTTG, 483ProF, GCACCCAGAGTTTCTACCCTCCACCAGTCCC, 483ProR, GGGACTGGTGGAGGGTAGAAACTCTGGGTGC, 491ArgF, GTCCCTTACTACCCAGGGACTATGAAGAGTTTG and 491ArgR, CAAACTCTTCATAGTCCCTGGGTAGTAAGGGAC. All the mutated GYS2 cDNAs were completely sequenced to ensure the presence of only the desired mutation. DNA for transfections was purified using the Wizard miniprep purification system (Promega). The concentration and purity of the DNA was determined spectrophotometrically and by an agarose gel electrophoresis.

\section{Expression of the mutated GSs and determination of the GS activity}

COS7 cells were grown in DME (Sigma Aldrich Sweden AB, Stockholm, Sweden), with $10 \%$ FBS, $2 \%$ penicillin/streptomycin, $1 \%$ L-glutamine, and trypsinized the day before transfections when $75 \%$ confluent. $6 \times 10^{5}$ cells were placed on $25-\mathrm{cm}^{2}$ plates and grown for $16 \mathrm{~h}$ to reach $75 \%$ confluency. $3 \mu \mathrm{g}$ of the GYS2WT-pcDNA3.1-, the mutated GYS2 cDNAs, or the pcDNA3.1 - vector only was mixed with $20 \mu \mathrm{l}$ of lipofectamine (Life Technologies, Gaithersburg, MD) and $800 \mu \mathrm{l}$ of DME and incubated at room temperature for $30 \mathrm{~min}$. The COS7 cells were rinsed with DME, $3.2 \mathrm{ml}$ DME was added to the DNA-lipofectamine mixtures, and this solution was pipetted on the cells. The cells were incubated in this solution for $5 \mathrm{~h}$ at $37^{\circ} \mathrm{C}$ with $10 \% \mathrm{CO}_{2}$. Thereafter, $4 \mathrm{ml}$ of DME with $20 \% \mathrm{FBS}, 4 \%$ penicillin/ streptomycin, and $2 \%$ L-glutamine was added and the incubation was continued for $40 \mathrm{~h}$. The cells were trypsinized, washed with $1 \times$ PBS, and stored at $-86^{\circ} \mathrm{C}$ until analyzed. Cells were homogenized in a ground-glass homogenizer as described earlier and the GS activity was determined at 0.3 and $7.1 \mathrm{mM}$ UDP glucose (UDPG) with 0.1 and $10 \mathrm{mM}$ glucose-6-phosphate (G-6-P) using a fluorometric method (20). GS activity was determined in duplicates from cell homogenates from three separate transfections. Protein amount was assayed according to Lowry et al. (21) and GS activity was given as nanomoles per minute per milligram of protein. GS activity at 7.1 $\mathrm{mM}$ UDPG with $10 \mathrm{mM} \mathrm{G-6-P}$ is used as an estimate of the total enzyme activity.

\section{Western blot analysis}

The expression of the GYS2-WT and the different mutated liver GSs was analyzed using Western blot analysis. A negative control (COS7 cells transfected with the pcDNA3.1 vector), GYS2-WT, GYS239Ser, GYS2-246X, GYS2-339Pro, GYS2-446Asp, GYS2-479Gln, GYS2-483Pro, GYS2-491Arg, and a rat liver control sample were homogenized in $50 \mathrm{mM} \mathrm{KF}, 10 \mathrm{mM}$ EDTA, pH 7.0. Samples were separated on $7.5 \%$ acrylamide resolving, $4.5 \%$ acrylamide stacking gels at $200 \mathrm{~V}$ for $45 \mathrm{~min}$. The proteins were electrotransferred to nitrocellulose membranes at $100 \mathrm{~V}$ for $60 \mathrm{~min}$ and incubated with a polyclonal rat liver GS antibody prepared in chicken (IgY). The second antibody was anti-chicken IgG (Sigma) and the detection was done using avidin alkaline phosphatase conjugate (Bio Rad, Hercules, CA), paranitroblue tetrazolium chloride, and 5-bromo-4-chloro-3-indolyl phosphate.

\section{GenBank accession numbers}

The nucleotide sequences of the GYS2 gene were submitted to the EMBL with accession numbers AJ003087-AJ003102.

\section{Results}

Clinical and biochemical phenotype. Fasting hypoglycemia associated with increased plasma concentrations of $\beta$-hydroxybutyrate and free fatty acids dominated the clinical picture in six of the children with GSD-0. After a glucose challenge or a meal, the plasma concentrations of ketones and free fatty acids were normalized while plasma glucose and lactate concentrations rose markedly. In the five children who had liver biopsies, the liver glycogen content was low, whereas the liver GS activity was low or nil (Table I). Glycogen structure, where analyzed, was normal (Table I).

Genomic structure of the GYS2 gene. Cloning and characterization of the five genomic clones isolated with $G Y S 2$ cDNA probes revealed that the GYS2 gene is composed of 16 exons and spans $>30 \mathrm{~kb}$ (Table III and see Fig. 2). All the sequences at the exon-intron junctions conform to the GT/AG rule (22) (Table III) and the exon-intron boundaries are conserved with the human muscle GS gene (GYS1) (23). The genomic clone GYS2- $\lambda$ E1-3, which was extracted with the 441-bp PCR probe, was shown to contain exon 1 and the 5 -flanking region of the GYS2 gene. The genomic clone GYS2- $\lambda 21$ was found to contain exons $2-4$, the GYS2- $\lambda 14$ exons $2-5$, the GYS $2-\lambda 6$ exons $6-11$, and the GYS2- $\lambda 16$ exons $12-16$. None of the extracted genomic clones contained the whole intron 1 or intron 11 sequences, and because we could not amplify these introns from a genomic DNA, they are presumed to be long.

Linkage analysis. The segregation of chromosome 12p12.2 markers in five families with GSD-0 is shown in Fig. 1. Using a recessive model of inheritance, linkage of GSD-0 to the GYS2 gene was suggested in these families with a combined LOD score of $2.9(P<0.01)$.

Mutation screening. Mutation screening by SSCP and sequencing revealed several DNA variants in the affected children (Fig. 1). In family Y, the proband (patient 1), whose both alleles had similar haplotypes, carried a mutation causing a premature stop codon in exon 5, Arg246X (CGA $\rightarrow$ TGA), on both alleles (Fig. 1). Also two of the unaffected brothers of patient 1 carried the Arg246X mutation on one allele, whereas the third unaffected brother did not have the mutation. In family $M$ (patients 2-4), a mutation in the $5^{\prime}$-donor splicing site of intron $6\left(\mathrm{G}^{+1} \mathrm{~T} \rightarrow \mathrm{CT}\right)$ was identified. The mutation was inherited from the mother by all three affected children. The DNA 
Table III. Exon-Intron Boundary Sequences of the GYS2 Gene

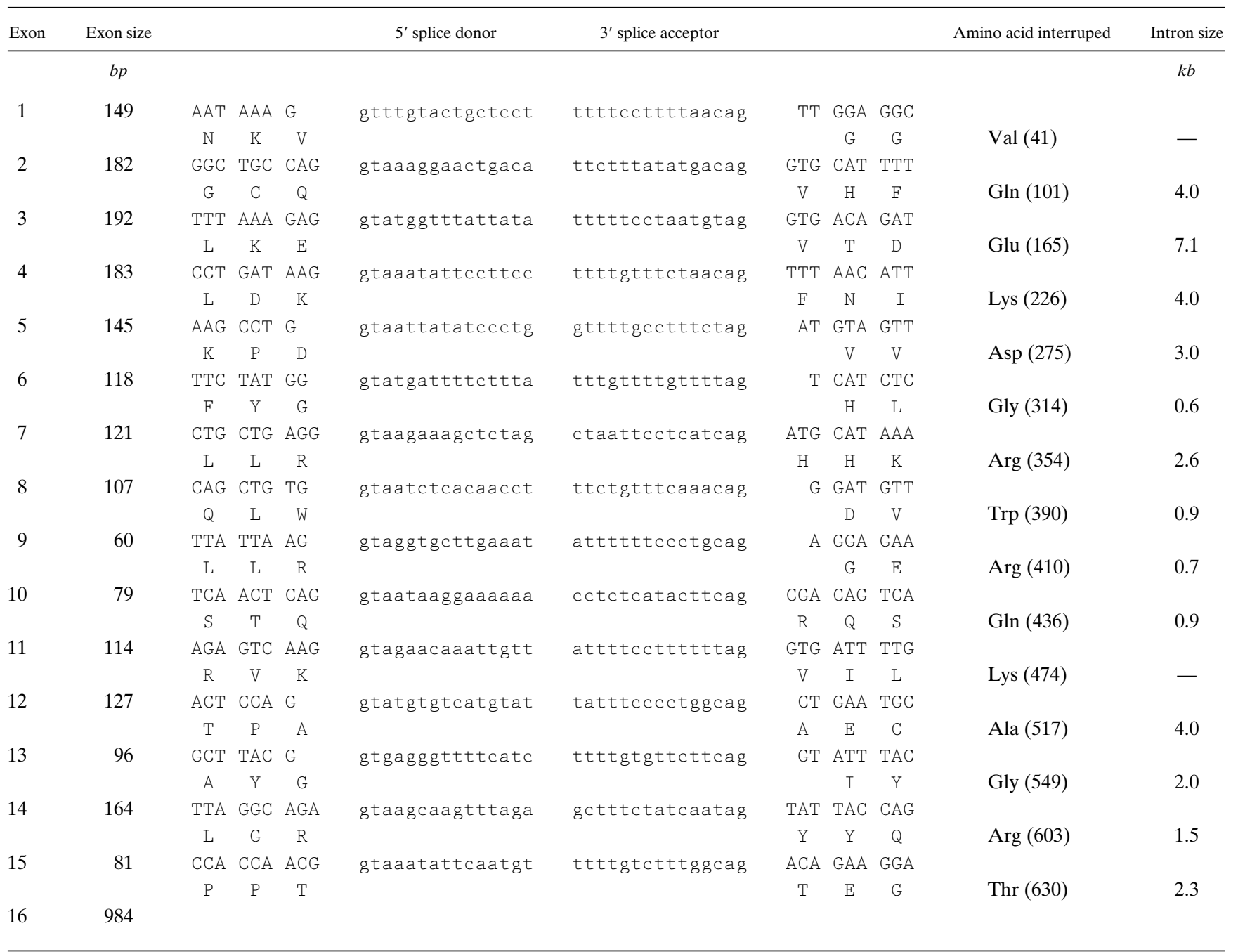

Exon sequences are in capital letters and intron sequences are in lowercase letters. The amino acids corresponding to the exon sequence are shown under the nucleotide sequence, and the amino acid preceding or interrupted by an intron is indicated. Introns are positioned by applying the GT/AG rule (22). The sizes of introns 1 and 11 were not determined.

from the father was not available for the study but a Pro479Gin mutation (CCA $\rightarrow$ CAA) in exon 12 was identified in all three children. Since this mutation occurred on identical haplotypes and was not seen in the mother, it most likely represents the mutation inherited from the father.

In family $\mathrm{F}$, patient 5 was shown to carry two missense mutations. A Ala339Pro mutation (GCT $\rightarrow$ CCT) in exon 7 was inherited from the mother and a Met491Arg mutation (ATG $\rightarrow$ AGG) in exon 12 was inherited from the father. Also, the unaffected sister carried the Ala339Pro mutation, but she did not have the Met491Arg mutation. In family J, patients 6 and 7 carried a Asn39Ser mutation $(\mathrm{AAT} \rightarrow \mathrm{AGT})$ in exon 1 and a Ser483Pro mutation (TCC $\rightarrow \mathrm{CCC}$ ) in exon 12 . In family $\mathrm{S}$, a His446Asp mutation (CAC $\rightarrow$ GAC) in exon 11 was inherited from the mother by patients 8 and 9 , but no mutation that could have been inherited from the father was found despite SSCP analysis and direct sequencing of all the exons and 450 bp of the $5^{\prime}$-flanking region of the GYS2 gene. None of the identified mutations could be seen in the 400 control chromo- somes. All the mutations identified in the GYS2 gene are summarized in Fig. 2.

Expression of the mutated liver GS enzymes in the COS7 cells. The expression of the wild-type liver GS in COS7 cells resulted in $\sim 30$ times higher total GS activity compared with intracellular GS activity $(334 \pm 13$ vs. $11 \pm 4 \mathrm{nmol} / \mathrm{min} \times \mathrm{mg}$ protein) (Table IV). The GS activities of the mutated liver GS enzymes were at highest 3.5 and $1.1 \%$ of the wild-type activity (Table IV). Western blot analysis using a polyclonal rat liver GS antibody detected the expected $80-\mathrm{kD}$ band in the GYS2WT, GYS2-39S, GYS2-446D, GYS2-479Q, GYS2-483P, and GYS2-491R and in the rat liver sample. The antibody did not detect the GYS2-246X and GYS2-339Pro samples (data not shown).

\section{Discussion}

Knowledge of the genomic structure allowed us to screen the GYS2 gene in children with proven or suspected GSD-0. A 

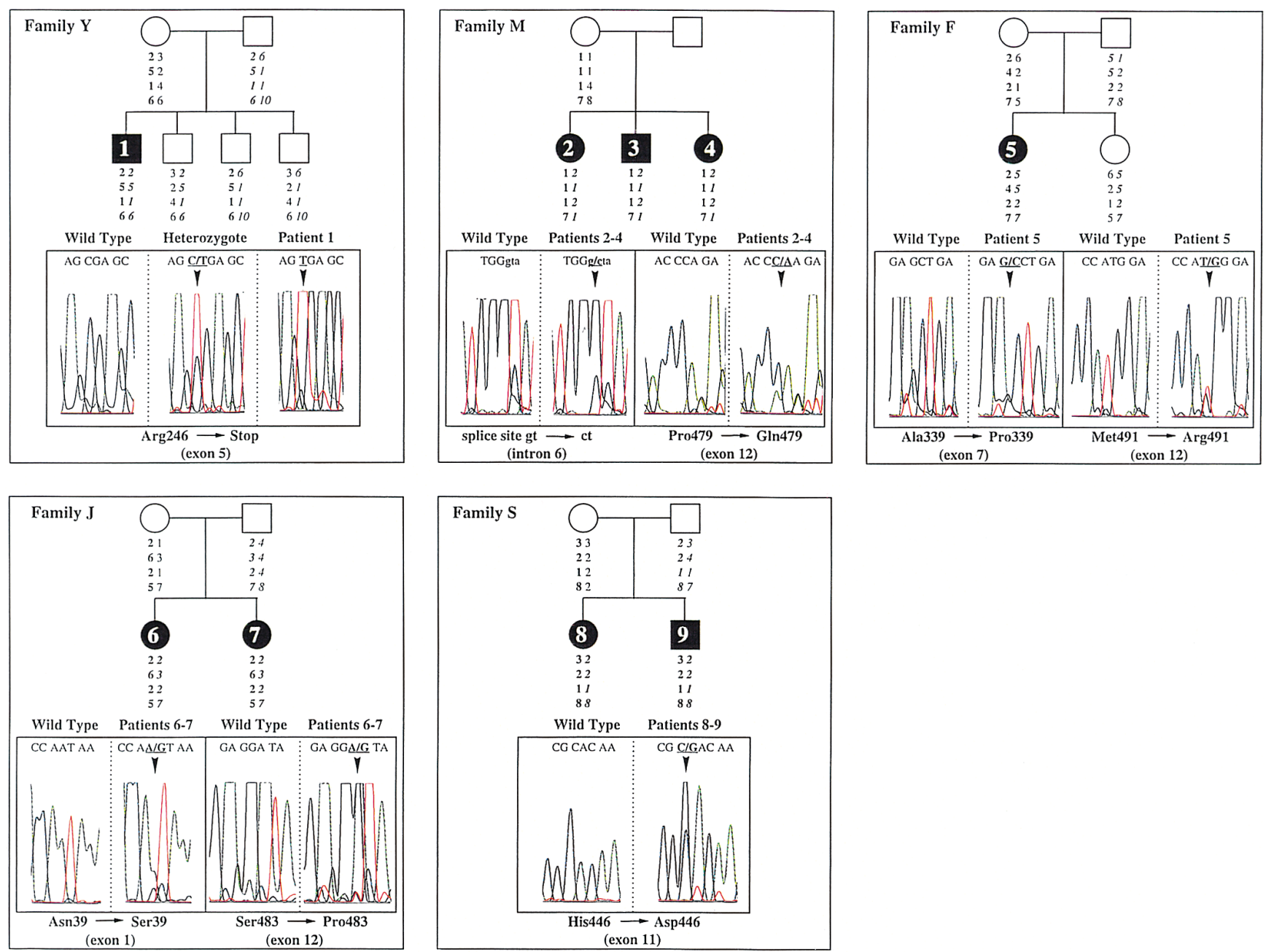

Figure 1. Pedigrees of the patients with GSD-0, the segregation of GSD-0 and chromosome 12p12.2 markers, and the mutations identified in the GYS2 gene in the families. The patient numbers are indicated inside the symbols. The haplotypes (markers GYS2-CA, GATAglHO1, D12S1606, and D12S1688, respectively) are shown under the symbols. The paternal haplotypes are shown in cursive and the affected chromosomes are in bold. The nucleotide sequences and the plots from the automatic sequence analysis are illustrated under the corresponding pedigrees. The plots show the comparison of the wild-type sequences (sequenced from the parent not carrying this mutation) and the mutated sequences (sequenced from the patients). In family Y, sequence results are shown from unaffected sibling without the mutation (wild-type), from the mother (heterozygote), and from patient 1 , who carries the mutation on both chromosomes. All of the sequences are illustrated as sense sequences except for the Ser483Pro mutation in family $\mathrm{J}$, for which the antisense sequence is shown. The mutated sequences are underlined, the mutations are indicated with arrows, and the resulting amino acid changes are shown under the sequences.

suggestive recessive linkage to the GYS2 gene was established in these five families with nine affected children and mutation screening of the GYS2 gene revealed unique mutations in each family (Figs. 1 and 2).

In family Y, patient 1 carried an Arg246X nonsense mutation in exon 5 on both alleles (Fig. 1). This premature stop codon presumably leads to loss of $65 \%$ of the $\mathrm{COOH}$-terminal part of the protein, including amino acids 246-400 and 500600 , which may include the catalytic and the allosteric G-6-P binding sites (15). Surprisingly, this patient had practically normal glycogen concentration and the least severe reduction in the GS activity (Table I). His unrelated parents and two brothers, who were heterozygous carriers of the Arg246X mutation, did not show ketotic fasting hypoglycemia nor an exaggerated rise in blood glucose and lactate during the oral glucose tolerance test. However, liver biopsies have not been performed in them. Expression of the GYS2 cDNA with the Arg246X mutation in COS cells demonstrated that this stop codon leads to a total loss of GS activity (Table IV). Rat liver GS antibody did not bind to the GYS2-246X in the Western blot analysis, which indicates that this truncated GS was either not recognized by the antibody or the expressed protein is unstable.

In patients $2-4$ in family $M$ we identified a mutation in the 5 -donor splicing site of intron 6 , which was inherited from the mother by all three affected children (Fig. 1). This mutation destroys the conserved donor splice site, which predictably results in skipping of exon 6 (amino acids 275-314) and a frameshift with exons 5 and 7 linked together. This, in turn, will create a premature stop codon in exon 7. If expressed, this transcript would code for a $57 \%$ truncated protein with 29 miscoded amino acids in the $\mathrm{COOH}$-terminal end. The mother, who carries the mutation only in one chromosome, also devel- 


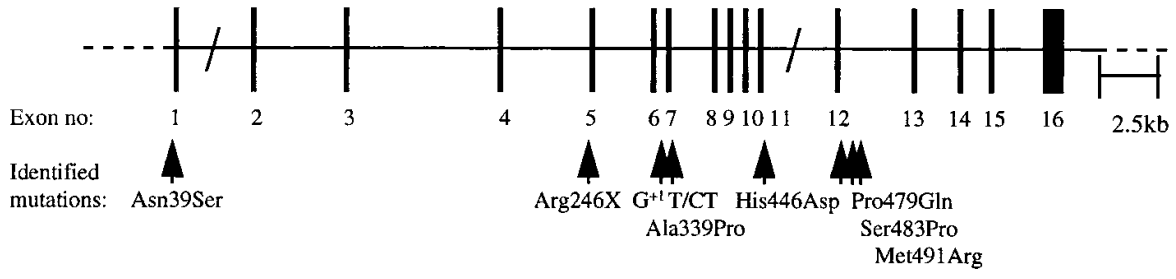

Figure 2. Human GYS2 gene map. Each exon is represented by a box and numbered. The locations of identified mutations in families with GSD-0 are indicated by arrows. ops hypoglycemia during prolonged fasting, which could indicate that this truncated protein may disturb the structure of the wild-type liver GS, which is believed to be a dimer (24). The DNA from the father was not available but a Pro479Gln mutation in exon 12 was identified in all three children. Since this mutation occurred on identical haplotypes and was not seen in the mother, it most likely represents the mutation inherited from the father. In agreement with this, no GS activity was detected in COS cells expressing the mutated enzyme with glutamine in codon 479 instead of proline (Table IV).

Five different missense mutations in exons 1 (Asn39Ser), 7 (Ala339Pro), 11 (His446Asp), and 12 (Ser483Pro and Met491Arg) were identified in patients 5-9 from families F, J, and $\mathrm{S}$ (Fig. 1). In families $\mathrm{F}$ and $\mathrm{J}$, the patients were compound heterozygotes for two different mutations on different alleles. When the mutated GYS 2 cDNAs were expressed in COS cells, the GS activities at high UDPG and G-6-P concentrations were at highest 3.5 and $1.1 \%$ of the wild-type activity (Table IV). All the expressed GSs with missense mutations, except the GYS2-339Pro, were detected as $80-\mathrm{kD}$ bands in the Western blot analysis, showing that these mutations affect the GS activity. Whether the GYS2-339Pro was not detected because of structural changes leading to loss of epitopes detected by the used antibody or if this mutation affects the stability of the mutated enzyme is not known. In family S (patients 8 and 9), only one mutation was identified despite SSCP analysis and direct sequencing of all the exons and $450 \mathrm{bp}$ of the $5^{\prime}$-flanking region. The unidentified disease-causing mutation in family $S$ is presumably located outside the coding region and exon-

Table IV. Expression of the Mutated Glycogen Synthases in COS Cells

\begin{tabular}{lcc}
\hline Transfected DNA & GS activity & Total GS activity \\
\hline & nmol/min $\times$ mg protein & nmol/min $\times$ mg protein \\
pcDNA3.1-* & $5.1 \pm 3.9$ & $11.1 \pm 3.9$ \\
GYS2-WT & $33.4 \pm 0.7$ & $333.7 \pm 12.9$ \\
GYS2-39Ser & $-3.8 \pm 0.5$ & $11.8 \pm 1.2$ \\
GYS2-246X & $-6.3 \pm 1.3$ & $-2.7 \pm 1.1$ \\
GYS2-339Pro & $-4.6 \pm 0.4$ & $3.8 \pm 0.6$ \\
GYS2-446Asp & $-4.5 \pm 0.9$ & $0.0 \pm 2.5$ \\
GYS2-479Gln & $-3.0 \pm 0.3$ & $-3.0 \pm 3.0$ \\
GYS2-483Pro & $-5.8 \pm 0.3$ & $-4.1 \pm 0.7$ \\
GYS2-491Arg & $-7.2 \pm 0.6$ & $-4.8 \pm 0.3$
\end{tabular}

Data are means \pm SD. $*$ The negative control giving an estimation of the intracellular GS activity. Intracellular GS activity has been subtracted from the other results. ${ }^{\ddagger} \mathrm{GS}$ activity at $7.1 \mathrm{mM}$ UDPG with $0.1 \mathrm{mM} \mathrm{G}-6-P$. ${ }^{8}$ GS activity at $7.1 \mathrm{mM}$ UDPG with $10 \mathrm{mM}$ G-6-P. intron junctions and could, for example, silence or impair the expression of the GYS2 gene or produce novel splice sites altering the mRNA processing.

We think that the identified mutations are the cause of GSD-0 for the following reasons. First, we expressed the mutated GSs in COS cells and demonstrated that all of these mutations result in either immeasurable or very low GS activity, regardless of whether measured as total activity or at physiological concentrations of UDPG or G-6-P (Table IV). Second, seven of the affected children carried mutations in both alleles. Finally, the mutations were not detected in 400 control chromosomes. We can only speculate about the mechanisms by which the identified mutations could affect the catalytic efficiency of the enzyme. The Asn39Ser mutation is located close to the proposed UDPG binding site (amino acids 40-43) (25, 26). The Ala339Pro mutation in the highly conserved exon 7 could result in distortion of the three-dimensional structure by introducing a helix breaking amino acid proline. The His446Asp mutation in exon 11 represents a change from a reactive basic to an acidic amino acid and could change the angle beyond the conserved region coded by exon 12 . This, in turn, could affect transmission of the phosphorylation signal to the catalytic and/or G-6-P binding site. The Pro479Gin, Ser483Pro, and Met491Arg mutations are located in exon 12, which shows $95 \%$ conservation between the GYS2 and GYS1 genes $(15,23)$. These substitutions occur in an area of strong negative charge density and thus likely affect the regulation of the catalytic efficiency and/or transmission of phosphorylation signals to the G-6-P binding site (27).

Some intriguing findings remain to be explained. Although the GS activity was almost lacking in the liver of the patients, the glycogen content was only moderately decreased and the glycogen structure was apparently normal (Table I, reference 3 ). This points at an alternative, GS-independent pathway for glycogen synthesis in the liver (28). The most likely explanation would be the reversal of the glycogen phosphorylase reaction. Already Lewis et al. (1) suggested that under certain circumstances the function of liver GS could be taken up by glycogen phosphorylase, as the phosphorylase reaction is reversible and phosphorylase activity exceeds synthase activity by 10 -fold in the liver (29). Even a modest reversal of phosphorylase activity could thus result in significant glycogen synthesis. However, this would require a marked decrease in cell $\mathrm{pH}$ and a very high glucose-1-phosphate concentration (30).

Fasting hypoglycemia is usually the consequence of insufficient liver glucose output, either due to inappropriately high insulin secretion or inherited defects in liver glucose production. The latter represents the sum of two processes, glycogenolysis and gluconeogenesis. In overnight fasted healthy individuals the two processes contribute equally to net liver glucose output, whereas during prolonged fasting the contribu- 
tion of gluconeogenesis increases $(31,32)$. Although some glycogen was shown to be synthesized in the liver of GSD-0 patients, they become hypoglycemic already a few hours after the meal. A recent report by Petersen et al. may have illuminated this problem by showing that during hypoglucagonemic euglycemic hyperinsulinemia, the inhibition of net glycogenolysis in the liver is exclusively through the activation of GS, whereas glycogen phosphorylase is unaltered, resulting in extensive glycogen cycling (33). GS is not activated in the liver of GSD-0 patients and the glycogen stores are most likely depleted during normoglycemic conditions. How a defect in liver glycogen synthesis without a concomitant defect in gluconeogenesis can lead to hypoglycemia remains unclear. Metabolic profiles $(2,3$, 10) suggest that the patients cannot switch to gluconeogenesis rapidly enough to ensure a normal hepatic glucose output (11). It is possible that glucagon is suppressed by the postprandial hyperglycemia characteristic of the patients with GSD-0 and that the glucagon to insulin ratio remains too low to stimulate phosphoenolpyruvate carboxykinase, the rate-limiting enzyme for gluconeogenesis (34). The postprandial hyperglycemia and hyperlactatemia is explained by an inability to trap sufficient glucose as glycogen in the liver (11).

Although GSD-0 has been considered a rare disorder, it may be an underdiagnosed disease (3). In four families, five affected siblings were discovered only after the probands had been diagnosed $(3,12)$. The disease can obviously have a mild course. The distinction from other forms of ketotic hypoglycemia in childhood is difficult. The availability of a genetic diagnosis of the disease should now allow screening of symptomatic children.

Glucose intolerance and postprandial hyperglycemia were consistent findings in the patients. Therefore, the question arises of whether mutations in the GYS2 gene could contribute to glucose intolerance of type 2 diabetes as well. When screening 165 patients with type 2 diabetes and 165 healthy controls, we did not find any association between the GYS2-CA microsatellite polymorphism and type 2 diabetes. Neither could we observe linkage to this chromosomal region in families with type 2 diabetes (35).

In conclusion, mutations in the GYS2 gene provide a genetic explanation for GSD-0. The disease certainly involves a defect in the liver GS and we propose that the name GSD-0 is replaced by liver GS deficiency. Glycogen biogenesis is still not fully understood (36). The findings of only moderately decreased liver glycogen stores may point at an alternative, GSindependent pathway for glycogen formation in the liver.

\section{Acknowledgments}

We are indebted to the families for their participation, to Dr. L.K. Fisher for referring patients 2-4, to Dr. Y.T. Chen for performing the liver biopsy analyses of patient 2, and to Ms. M. Åberg for excellent technical assistance.

This work was supported by grants from the Sigrid Juselius Foundation (L.C. Groop), the Albert Påhlssons Foundation (M. Orho), the Swedish Medical Research Council (L.C. Groop and M. Orho), Medical Faculty of Lund University (M. Orho), Malmö University Hospital (M. Orho), Ernhold Lundströms Foundation (M. Orho), Novo Nordic Foundation (L.C. Groop), EEC grant BMH4-CT950662 (L.C. Groop), ROI DK 43018 from the National Institutes of Health (M.C. Gannon), and Merit Review Research Funds from the Department of Veterans Affairs (F.Q. Nuttall).

\section{References}

1. Lewis, G., J. Spencer-Peet, and K. Stewart. 1963. Infantile hypoglycemia due to inherited deficiency of glycogen synthase in the liver. Arch. Dis. Child. 38:40-48.

2. Aynsley-Green, A., D.H. Williamson, and R. Gitzelmann. 1977. Hepatic glycogen synthetase deficiency. Definition of syndrome from metabolic and enzyme studies on a 9-year-old girl. Arch. Dis. Child. 52:573-579.

3. Gitzelmann, R., M.A. Spycher, G. Feil, J. Muller, B. Seilnacht, M. Stahl, and N.U. Bosshard. 1996. Liver glycogen synthase deficiency: a rarely diagnosed entity. Eur. J. Pediatr. 155:561-567.

4. McKusick, V.A. 1998. On Line Mendelian Inheritance in Man. URL: http://www3.ncbi.nlm.nih.gov/htbin-post/Omim/dispmim?240600

5. Cao, Z., R. Umek, and S.L. McKnight. 1991. Regulated expression of C/EBP isoforms during adipocyte conversion of 3T3-L1 cells. Genes Dev. 5: 1538-1552.

6. Williams, S., C. Cantwell, and P. Johnson. 1991. A family of C/EBPrelated proteins capable of forming covalently linked leucine zipper dimers in vitro. Genes Dev. 5:1553-1567.

7. Darlington, G., N. Wang, and R. Hanson. 1995. A critical regulator of genes governing integrative metabolic processes. Curr. Opin. Gen. Dev. 5:565570 .

8. Wang, N.D., M.J. Finegold, A. Bradley, C.N. Ou, S.V. Abdelsayed, M.D. Wilde, L.R. Taylor, D.R. Wilson, and G.J. Darlington. 1995. Impaired energy homeostasis in C/EBP alpha knockout mice. Science. 269:1108-1112.

9. Nuttall, F.Q., M.C. Gannon, V.L. Kubic, and K.J. Hoyt. 1994. The human liver glycogen synthase isozyme gene is located on the short arm of chromosome 12. Genomics. 19:404-405.

10. Aynsley-Green, A., D.H. Williamson, and R. Gitzelmann. 1977. The dietary treatment of hepatic glycogen synthetase deficiency. Helv. Paediatr. Acta. 32:71-75.

11. Byrne, B.M., M.D. Gillmer, R.C. Turner, and A. Aynsley-Green. 1995. Glucose homeostasis in adulthood and in pregnancy in a patient with hepatic glycogen synthetase deficiency. Br. J. Obstet. Gynaecol. 102:931-933.

12. Aynsley-Green, A., D.H. Williamson, and R. Gitzelmann. 1978. Asymptomatic hepatic glycogen-synthetase deficiency [letter]. Lancet. 1:147-148.

13. Hers, H.G. 1964. Glycogen storage disease. In Advances in Metabolic Disorders. Vol. 1. R. Levine and R. Luft, editors. Academic Press, New York. $1-44$.

14. Krisman, C.R. 1962. A method for the calorimetric estimation of glycogen with iodine. Anal. Biochem. 4:17-23.

15. Nuttall, F.Q., M.C. Gannon, G. Bai, and E.Y. Lee. 1994. Primary structure of human liver glycogen synthase deduced by cDNA cloning. Arch. Biochem. Biophys. 311:443-449.

16. Gambino, V., S. Menzel, J.B. Trabb, K.S. Xiang, T. Lindner, A. Louit, E. Chen, L.E. Mereu, H. Furuta, N. Iwasaki, et al. 1996. An approach for identifying simple sequence repeat DNA polymorphisms near cloned cDNAs and genes. Linkage studies of the islet amyloid polypeptide/amylin and liver glycogen synthase genes and NIDDM. Diabetes. 45:291-294.

17. The Genome Database. 1997. URL:http://gdbwww.gdb.org/.

18. Kruglyak, L., M.J. Daly, M.P. Reeve-Daly, and E.S. Lander. 1996. Parametric and nonparametric linkage analysis: a unified multipoint approach. Am. J. Hum. Genet. 58:1347-1363.

19. Orita, M., Y. Suzuki, T. Sekiya, and K. Hayashi. 1989. Rapid and sensitive detection of point mutations and DNA polymorphisms using the polymerase chain reaction. Genomics. 5:874-879.

20. Schalin-Jäntti, C., M. Härkönen, and L. Groop. 1992. Impaired activation of glycogen synthase in people at increased risk for developing NIDDM. Diabetes. 41:598-604.

21. Lowry, O., N. Rosebrough, A. Farr, and R. Randall. 1951. Protein measurement with the Folin phenol reagent. J. Biol. Chem. 193:265-275.

22. Breathnach, R., and P. Chambon. 1981. Organization and expression of eucaryotic split genes coding for proteins. Annu. Rev. Biochem. 50:349-383.

23. Orho, M., P. Nikula-Ijäs, C. Schalin-Jäntti, M.A. Permutt, and L.C. Groop. 1995. Isolation and characterization of the human muscle glycogen synthase gene. Diabetes. 44:1099-1105.

24. Roach, P.J. 1986. Liver Glycogen Synthase. Academic Press, Orlando, FL. 499-539.

25. Mahrenholz, M.M., Y. Wang, and P.J. Roach. 1988. Catalytic site of rabbit glycogen synthase isoenzymes. Identification of an active site lysine close to the amino terminus of the subunit. J. Biol. Chem. 263:10561-10567.

26. Furukawa, K., M. Tagaya, K. Tanizawa, and T. Fukui. 1993. Role of the conserved Lys-X-Gly-Gly sequence at the ADP-glucose binding site in Escherichia coli glycogen synthase. J. Biol. Chem. 268:23837-23842.

27. Tan, A.W., and F.Q. Nuttall. 1983. Endogenous phosphates on liver glycogen synthase D and synthase I. Studies on the number and location. J. Biol. Chem. 258:9624-9630.

28. Nuttall, F.Q., and M.C. Gannon. 1993. Allosteric regulation of glycogen synthase in liver. A physiological dilemma. J. Biol. Chem. 268:13286-13290.

29. Nuttall, F.Q., D.P. Gilboe, M.C. Gannon, C.B. Niewoehner, and A.W.H. Tan. 1988. Regulation of glycogen synthesis in the liver. Am. J. Med. $85: 77-85$. 
30. Ercan, N., M.C. Gannon, and F.Q. Nuttall. 1996. Allosteric regulation of liver phosphorylase a: revisited under approximated physiological conditions. Arch. Biochem. Biophys. 328:255-264.

31. Rothman, D.L., I. Magnusson, L.D. Katz, and G.I. Shulman. 1991. Quantitation of hepatic glycogenolysis and gluconeogenesis in fasting humans with 13C NMR. Science. 254:573-576.

32. Landau, B.R., J. Wahren, V. Chandramouli, W.C. Shumann, K. Ekberg, and S.C. Kalhan. 1996. Contributions of gluconeogenesis to glucose production in the fasted state. J. Clin. Invest. 98:378-385.

33. Petersen, K.F., D. Laurent, D.L. Rothman, G.W. Cline, and G.I. Shulman. 1998. Mechanisms by which glucose and insulin inhibit net hepatic glycogenolysis in humans. J. Clin. Invest. 101:1203-1209.
34. Sasaki, K., T.P. Cripe, S.R. Koch, T.L. Andreone, D.D. Petersen, E.G. Beale, and D.K. Granner. 1984. Multi-hormonal regulation of phosphoenolpyruvate carboxykinase gene transcription: dominant role of insulin. $J$. Biol. Chem. 259:15242-15251.

35. Mahtani, M.M., E. Widen, M. Lehto, J. Thomas, M. McCarthy, J. Brayer, B. Bryant, G. Chan, M. Daly, C. Forsblom, et al. 1996. Mapping of a gene for type 2 diabetes associated with an insulin secretion defect by a genome scan in Finnish families. Nat. Genet. 14:90-94.

36. Alonso, M.D., J. Lomako, W.M. Lomako, and W.J. Whelan. 1995. A new look at the biogenesis of glycogen. FASEB (Fed. Am. Soc. Exp. Biol.) J. 9:1126-1137. 